\title{
Revealing the Neuroendocrine Response After Remoxipride Treatment Using Multi-Biomarker Discovery and Quantifying It by PK/PD Modeling
}

\author{
Willem J. van den Brink, ${ }^{1}$ Yin C. Wong, ${ }^{1}$ Berfin Gülave, ${ }^{1}$ \\ Piet H. van der Graaf, ${ }^{1,2}$ and Elizatbeth C. M. de Lange ${ }^{1,3}$
}

Received 29 June 2016; accepted 3 October 2016; published online 26 October 2016

\begin{abstract}
To reveal unknown and potentially important mechanisms of drug action, multibiomarker discovery approaches are increasingly used. Time-course relationships between drug action and multi-biomarker profiles, however, are typically missing, while such relationships will provide increased insight in the underlying body processes. The aim of this study was to investigate the effect of the dopamine D2 antagonist remoxipride on the neuroendocrine system. Different doses of remoxipride $(0,0.7,5.2$, or $14 \mathrm{mg} / \mathrm{kg})$ were administered to rats by intravenous infusion. Serial brain extracellular fluid (brainECF) and plasma samples were collected and analyzed for remoxipride pharmacokinetics (PK). Plasma samples were analyzed for concentrations of the eight pituitary-related hormones as a function of time. A Mann-Whitney test was used to identify the responding hormones, which were further analyzed by pharmacokinetic/ pharmacodynamic (PK/PD) modeling. A three-compartment PK model adequately described remoxipride PK in plasma and brainECF. Not only plasma PRL, but also adrenocorticotrophic hormone $(\mathrm{ACTH})$ concentrations were increased, the latter especially at higher concentrations of remoxipride. Brain-derived neurotropic factor (BDNF), follicle stimulating hormone (FSH), growth hormone $(\mathrm{GH})$, luteinizing hormone $(\mathrm{LH})$, and thyroid stimulating hormones $(\mathrm{TSH})$ did not respond to remoxipride at the tested doses, while oxytocin (OXT) measurements were below limit of quantification. Precursor pool models were linked to brainECF remoxipride PK by $\mathrm{E}_{\max }$ drug effect models, which could accurately describe the PRL and ACTH responses. To conclude, this study shows how a multi-biomarker identification approach combined with PK/PD modeling can reveal and quantify a neuroendocrine multi-biomarker response for single drug action.
\end{abstract}

KEY WORDS: blood-brain barrier; CNS; dose-response; hormones; pharmacokinetic/ pharmacodynamic models.

Electronic supplementary material The online version of this article (doi:10.1208/s12248-016-0002-3) contains supplementary material, which is available to authorized users.

\footnotetext{
${ }^{1}$ Systems Pharmacology, Division of Pharmacology, Leiden Academic Center for Drug Research, Leiden University, PO box 9502, 2300 RA, Leiden, The Netherlands.

${ }^{2}$ Certara QSP, Canterbury Innovation House, Canterbury, UK.

${ }^{3}$ To whom correspondence should be addressed. (e-mail: ecmdelange@lacdr.leidenuniv.nl)

Abbreviations ACTH, Adrenocorticotropic hormone; AL, Anterior lobe; BBB, Blood-brain barrier; BDNF, Brain-derived neurotropic factor; ECF, Extracellular fluid; FSH, Follicle stimulating hormone; $\mathrm{GH}$, Growth hormone; IL, Intermediate lobe; LH, Luteinizing hormone; ODE, Ordinary differential equation; OFV, Objective function value; OXT, Oxytocin; PACAP, Pituitary adenylate cyclase-activating polypeptide; PHDA, Periventricular hypothalamic dopaminergic; PK/PD, Pharmacokinetic/pharmacodynamic; PL, Posterior lobe; PRL, Prolactin; RSE, Relative standard error; THDA, Tuberohypothalamic dopaminergic; TIDA, Tuberoinfundibular dopaminergic; TSH, Thyroid stimulating hormone; VPC, Visual predictive check
}

\section{INTRODUCTION}

To better understand pharmacological effects of central nervous system (CNS) drugs on the whole biological system, including the unknown mechanisms of action, a holistic approach is key $(1,2)$. Unfortunately, the focus of current in vivo pharmacology is often on the known mechanism of action only (3). Therefore, to obtain insight in multiple system components, increasing efforts are made to show the utility of a multi-biomarker discovery approach, both in disease conditions and upon drug administration $(3,4)$. With that, the pathophysiological and pharmacological influences are reflected by a multi-biomarker response.

Thereby, it is not enough to investigate dose versus multi-biomarker response, because such relationships are not unique, i.e., condition dependent. It is therefore important to have insight into processes that govern drug distribution to target sites, target binding kinetics, signal transduction, and homeostatic feedback mechanisms. Such insight is obtained by multilevel studies, i.e., measurement of different 
biomarker types in a time-dependent manner and advanced pharmacokinetic/pharmacodynamic (PK/PD) modeling (5-7).

$\mathrm{PK} / \mathrm{PD}$ modeling was successfully applied for the selective dopamine D2 antagonist remoxipride, both in human (8) and rat (9), to predict the pharmacological response beyond the tested conditions. The pharmacological response was represented by prolactin (PRL), which is a well-known biomarker for D2 antagonism $(10,11)$. More specifically, dopamine binding to the D2 receptor inhibits the prolactin release from the pituitary into plasma, and therefore, D2 antagonism induces its release (10). Movin-Osswald and Hammarlund-Udenaes (1995) developed a PK/PD model to describe the PK of remoxipride in plasma in conjunction to its effect on the PRL kinetics (synthesis, release, and elimination) in human subjects, to successfully predict the PRL response after different and repeated doses. Then, in our lab, Stevens et al. (2012) developed a translational PK/PD model for remoxipride effects on PRL plasma concentrations in rats, using data on brain extracellular fluid (brainECF) concentrations that could be identified as target site concentrations. With that, they successfully predicted the human PK/PD data of remoxipride and PRL of Movin-Osswald and Hammarlund-Udenaes (1995). This indicated that plasma PRL is a translatable biomarker of D2 antagonism.

So far, D2 antagonism was only reflected by a single hormone, PRL. This hormone is part of the neuroendocrine system, which consists of the hypothalamus, the pituitary, and peripheral hormone glands (e.g., adrenal gland), containing a variety of hormones. The neuroendocrine hormones are highly regulated through feedback mechanisms of single hormones on their own secretion or that of others, both in a direct or an indirect manner. PRL and oxytocin (OXT), for example, interact through a positive feedback loop in female rats (12). Through the neuroendocrine system, the brain controls the plasma hormone levels in response to neurological stimuli. Thus, from a pharmacological perspective, a change in plasma hormone concentration may reflect a central drug action. This provides the unique opportunity to study central pharmacology on plasma hormone concentrations, overcoming the ethical and technical hurdles of taking samples from the human brain. Plasma hormones are released from the pituitary, which consists of the anterior lobe (AL), the intermediate lobe (IL), and the posterior lobe (PL). Dopaminergic neurons from the hypothalamus are involved in regulating all these parts of the pituitary (13). Release of hormones (e.g., PRL) that are stored in the AL may be regulated by dopamine that is secreted from tuberoinfundibular dopaminergic (TIDA) neurons into the portal vein. Hormones from the IL and the PL (e.g., OXT) are directly released, possibly from tuberohypothalamic dopaminergic (THDA) neurons that project into the pituitary. This tight connection between the dopaminergic and the neuroendocrine system inspired us to use the neuroendocrine system as a source for multi-biomarker discovery of dopaminergic agents.

Indeed, next to the dopamine-PRL connection, other interactions between the dopaminergic system and neuroendocrine hormones have also been reported. For example, the dopamine agonist bromocriptine is used to treat hypersecretion of adrenocorticotrophic hormone (ACTH) in Nelson's disease (14) as well as hypersecretion of growth hormone
$(\mathrm{GH})$ in acromegaly (15). Moreover, dopamine was found to inhibit secretion of GH from human pituitary cells (16), and dopamine D2 receptors have been identified on thyroid stimulating hormone (TSH) adenoma's (17). Furthermore, stimulation of the D2 receptor leads to suppression of the luteinizing hormone $(\mathrm{LH})$ by induction of the second messenger pituitary adenylate cyclaseactivating polypeptide (PACAP) in gonadotrophs (18). Therefore, to understand whether these interactions between the dopaminergic and neuroendocrine system are relevant to dopamine D2 antagonists in vivo, it seems of interest to investigate their broader neuroendocrine responses in a well-controlled animal study.

In this study, we applied a multi-biomarker discovery approach to investigate the neuroendocrine response to remoxipride in rats. Serial sampling of brainECF and blood was performed to determine the remoxipride PK in plasma and brain, following the procedures as earlier described (19). Blood samples were also analyzed for ACTH, brain-derived neurotropic factor (BDNF), follicle-stimulating hormone (FSH), GH, LH, OXT, PRL, and TSH to obtain a neuroendocrine multi-biomarker. We combined this approach with $\mathrm{PK} / \mathrm{PD}$ modeling to gain a comprehensive understanding of the PK/PD relation between remoxipride and the neuroendocrine system.

\section{METHODS}

\section{Animals}

All animal experiments were performed in accordance with the Dutch Law of Animal Experimentation. The study protocols (DEC14051/DEC13186) were approved by the Animal Ethics Committee in Leiden. Male Wistar rats $(n=111$, $264 \pm 17$ g), Charles River, The Netherlands) were housed in groups for 6-9 days until surgery (Animal Facilities Gorlaeus Laboratories, Leiden, The Netherlands), under standard environmental conditions with ad libitum access to food (Laboratory chow, Hope Farms, Woerden, The Netherlands) and acidified water. Artificial daylight was provided from 7:30 AM to 7:30 PM.

\section{Surgery}

Surgery was performed following the procedures as described earlier (19), with slight adaptations. In short, animals were kept under $2 \%$ isoflurane anesthesia while they underwent surgery. They received cannulas in the femoral artery for serial blood sampling and femoral vein for drug administration. A microdialysis guide (CMA 12 Elite PAES, Schoonebeek, The Netherlands) was implanted in caudateputamen (AP -1.0; L 3.0; V -3.4) for serial brainECF sampling. Twenty-four hours before the experiment, the microdialysis guide was replaced by a probe (CMA 12 Elite PAES, $4 \mathrm{~mm}$ polycarbonate membrane, cut-off $20 \mathrm{kDA}$, Schoonebeek, The Netherlands). Between surgery and experiments, the animals were kept individually in Makrolon type 3 cages for 7 days to recover from surgery. 


\section{Experiments}

Table I provides an overview of the groups and number of rats used (study numbers EW01 and WB02). All experiments started between 8:00 AM and 8:30 AM, with rats randomly assigned to receive $0,0.7,5.2$, or $14 \mathrm{mg} / \mathrm{kg}$ remoxipride by a 10 -min i.v. infusion at the start of experiment $(\mathrm{t}=0 \mathrm{~min})$. Microdialysate perfusion buffer was prepared as described earlier (20), and $60 \mathrm{~min}$ before the experiment, the perfusion was started using a flow rate of $1 \mu \mathrm{l} / \mathrm{min}$ until the end of experiment (see Table I for the sampling times). Samples with a deviated flow rate of $>10 \%$ were discarded. Microdialysate samples were stored at $4{ }^{\circ} \mathrm{C}$ during the experiment and at $-80^{\circ} \mathrm{C}$ after the experiment until analysis.

The extraction efficiency (in vivo recovery) of the microdialysis probe was determined following an in vivo loss experiment with 20, 100, 300, and $1000 \mathrm{ng} / \mathrm{ml}$ remoxipride. The microdialysate concentrations were corrected for an extraction efficiency of $11 \pm 0.5 \% \quad($ mean \pm SEM, $n=208)$. Blood samples of $200 \mu \mathrm{l}$ were taken at serial time points (Table I) through the arterial cannula and collected in heparin-coated eppendorf tubes. Animals received $200 \mu \mathrm{l}$ saline after each sampling. The samples were centrifuged (1000 rpm, $10 \mathrm{~min}$ ) for separation of plasma and were subsequently stored at $4^{\circ} \mathrm{C}$ during the experiment and at $-20^{\circ} \mathrm{C}$ after the experiment until analysis.

\section{Analytical Methods}

Remoxipride in Plasma and Microdialysates. Remoxipride concentrations in plasma and microdialysates were determined following a previously published liquid chromatography tandem mass spectrometry method (21) with small modifications to improve the column lifetime. In short, formic acid instead of trifluoro acetic acid was added to the solvents of on-line solid phase extraction and liquid chromatography, while maintaining the same performance in peak shape and resolution.

Plasma Hormones. The plasma hormone analysis was performed with Bio-Plex® ${ }^{\circledR}$ MAGPIX $^{\mathrm{TM}}$ technology (Biorad Laboratories, Veenendaal, The Netherlands). The hormones ACTH, BDNF, FSH, GH, LH, PRL, and TSH were analyzed using the commercially available multiplex assay for analysis of pituitary hormones (RTPMAG-86K, Rat pituitary magnetic bead panel, Merck Millipore, Darmstadt, Germany). We followed the protocol provided by the manufacturer, analyzing $10 \mu \mathrm{l}$ of each sample in duplo. OXT was analyzed using the commercially multiplex assay for analysis of neuropeptides (RMNPMAG-83K, Rat/mouse neuropeptide magnetic bead panel, Merck Millipore, Darmstadt, Germany). We followed the protocol provided by the manufacturer, analyzing $80 \mu \mathrm{l}$ of each sample. Data were acquainted, and concentrations were calculated using the Bio-Plex ${ }^{\circledR}$ Data Pro ${ }^{\text {TM }}$ software (Biorad Laboratories, Veenendaal, The Netherlands). Data below lower or above upper limit of quantifications were excluded, which was below $9 \%$ for all hormones, except for OXT for which most measurements were below limit of quantification $(<3.84 \mathrm{pg} / \mathrm{ml})$.

\section{Identification of Responding Hormones for Further PK/PD Analysis}

To select responding hormones, the plasma hormone concentration-time data were first corrected for individual hormone baseline concentrations as obtained before administration of remoxipride $(\mathrm{t}=-15 \mathrm{~min})$. These data were used to compare the experimental groups that received 5.2 or $14 \mathrm{mg} / \mathrm{kg}$ remoxipride with the placebo group (for baseline hormone values during the experimental period). A MannWhitney Wilcoxon non-parametric test was performed to compare the baseline corrected concentrations at each timepoint for each hormone. Hormones that showed a significant difference $(p<0.05)$ for at least one time-point were selected for further PK/PD analysis. All data analyses were performed using $\mathrm{R}$ version 3.1.1.

\section{PK/PD Modeling}

NONMEM® version 7.3.0 with subroutine ADVAN13 was used to perform the PK/PD modeling on the remoxipride concentrations in plasma and brainECF and the hormone concentrations in plasma. In addition to data from the studies described above, plasma and brainECF remoxipride concentrations were taken from a previously performed study in which 4,8 , and $16 \mathrm{mg} / \mathrm{kg}$ remoxipride was administered by a 30-min i.v. infusion (Table I) (22). Criteria to develop the best model were (i) significant drop in objective function value (OFV) calculated as -2 loglikelihood ratio $(>3.84, p<0.05$, $d f=1$ ); (ii) parameter precision; (iii) goodness-of-fit; (iv) correlations; (v) condition number; (vi) shrinkage; (vii) bootstrap; and (viii) visual predictive check (VPC). Furthermore, the PK/PD model for PRL was externally validated on available data from Stevens et al. 2012 (9).

A sequential PK/PD modeling approach was applied, in which the post hoc parameter estimates of the PK model were used as input for the PK/PD model. For the PK model, data were log-transformed, and an exponential error model was found to best describe the residual variation of the data, whereas for the PD models, a proportional error model was selected.

\section{RESULTS}

\section{Pharmacokinetics}

A three-compartment model was identified to describe the free remoxipride concentrations in plasma and brainECF (Fig. 1, middle part). Inclusion of saturable remoxipride clearance from plasma, described by Michaelis Menten kinetics, was found to improve the description of the data (dOFV -94, Fig. S1). This was particularly observed after low and high remoxipride doses (Fig. S1). Furthermore, in addition to passive blood-brain barrier (BBB) transport, distribution from brainECF to plasma (dOFV -238) or elimination from brainECF (dOFV -239) was found to improve the model fit. However, the model with distribution from brainECF to plasma showed imprecise parameter estimates (relative standard error (RSE) $>1000 \%$ ), and therefore, the model with elimination from brainECF was 


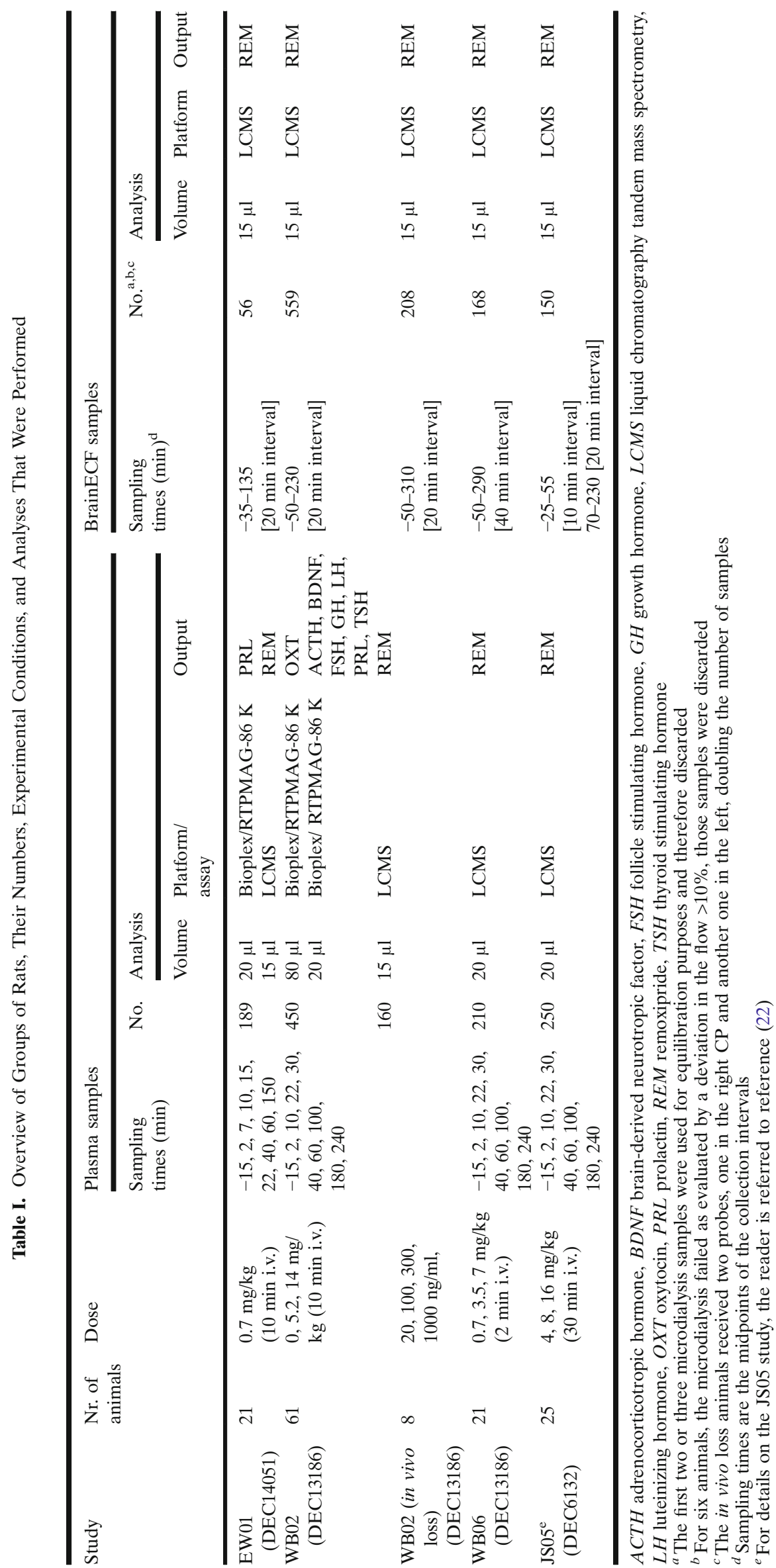




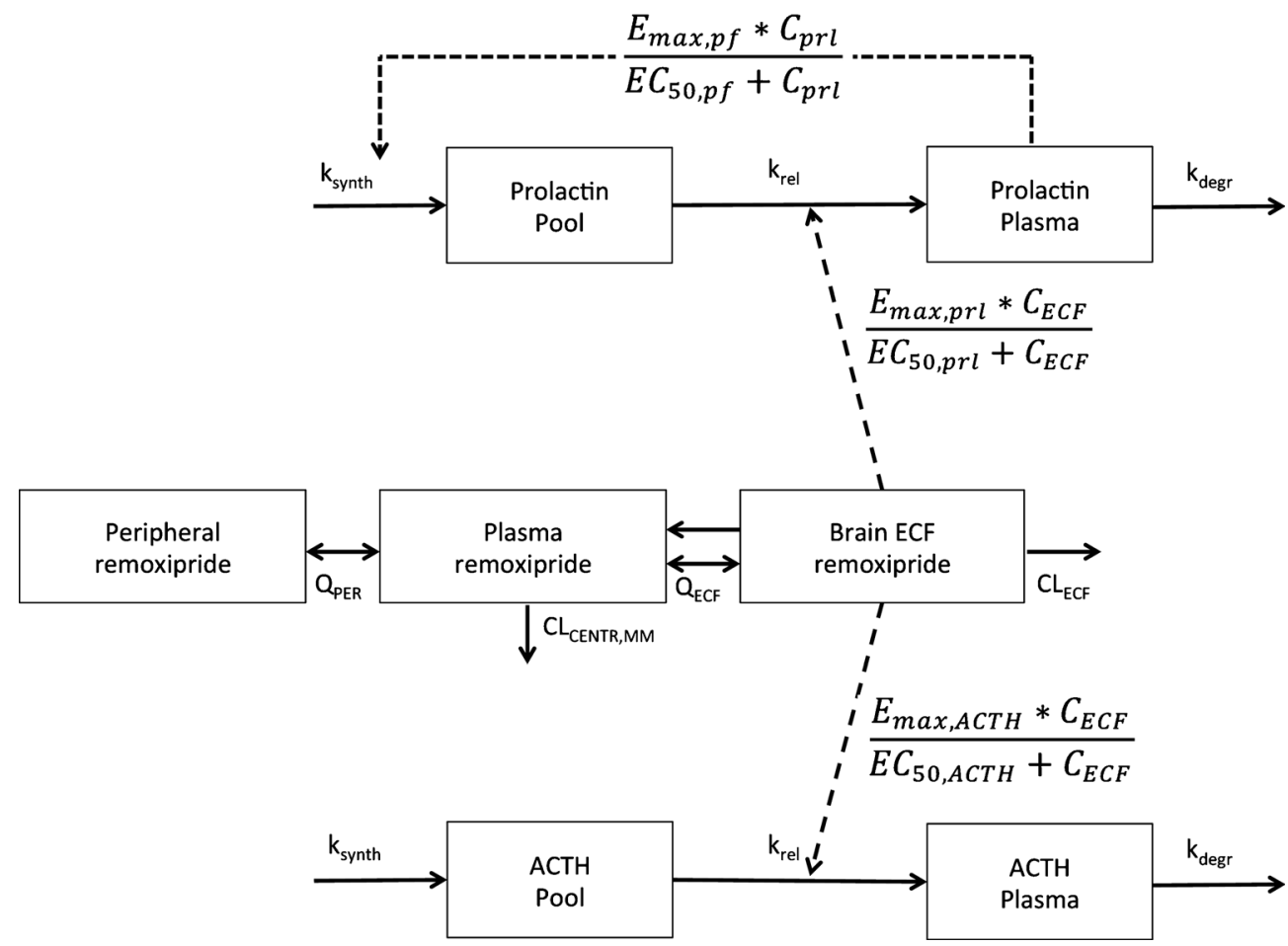

Fig. 1. Schematic overview of the final PK/PD model describing the pharmacokinetics of remoxipride in plasma and brain, as well as the pharmacodynamics for both PRL and ACTH. For explanation of the abbreviations, the reader is referred to Tables IV and V

selected. Parameter estimates showed good precision (RSE $<30 \%$ ), and bootstrap showed accurate estimates (Table II).

\section{Pharmacodynamics}

\section{Multi-Biomarker Discovery}

As shown in Fig. 2a, not only plasma PRL but also ACTH was found to respond to remoxipride treatment $(p<0.05)$. The other hormones (BDNF, FSH, GH, LH, and $\mathrm{TSH}$ ) did not show a significant response, while for oxytocin, most measurements were below limit of quantification. Plasma PRL showed a response after both 5.2 and $14 \mathrm{mg} /$ $\mathrm{kg}$, whereas ACTH only showed a response after $14 \mathrm{mg} / \mathrm{kg}$ remoxipride. The response time profiles of plasma PRL were similar for 5.2 and $14 \mathrm{mg} / \mathrm{kg}$ (Fig. 2b). Apparently, the response maximum was already reached after $5.2 \mathrm{mg} / \mathrm{kg}$. Therefore, an additional study was performed in which plasma PRL was measured after $0.7 \mathrm{mg} / \mathrm{kg}$ remoxipride (EW01 study, Table I). The plasma PRL concentrations after $0.7 \mathrm{mg} / \mathrm{kg}$ remoxipride were not only lower, but also exhibited a different longitudinal pattern. After 5.2 and $14 \mathrm{mg} / \mathrm{kg}$, but

Table II. Remoxipride Pharmacokinetics (PK), Parameter Estimates, and Bootstrap Results for the PK Model Following Different Doses of Remoxipride

\begin{tabular}{|c|c|c|c|c|}
\hline \multirow[t]{2}{*}{ Parameter } & \multirow[b]{2}{*}{ Parameter estimate } & \multirow[b]{2}{*}{ RSE (\%) } & \multicolumn{2}{|l|}{ Bootstrap $(n=50)$} \\
\hline & & & Bootstrap mean & CV (\%) \\
\hline $\mathrm{V}_{\text {max }, \text { centr }}(\mu \mathrm{M} / \mathrm{h})$ & 5.9 & 25 & 5.9 & 32 \\
\hline $\mathrm{k}_{\mathrm{m}, \mathrm{centr}}(\mu \mathrm{M})$ & 2.9 & 27 & 3.0 & 31 \\
\hline $\mathrm{V}_{\text {central }}(\mathrm{l})$ & 0.14 & 8 & 0.13 & 10 \\
\hline $\mathrm{Q}_{\text {PL-periph }}(\mathrm{l} / \mathrm{h})$ & 2.6 & 8 & 2.6 & 9 \\
\hline $\mathrm{V}_{\text {periph }}(\mathrm{l})$ & 0.52 & 15 & 0.52 & 14 \\
\hline $\mathrm{Q}_{\text {PL-ECF,passive }}(\mathrm{l} / \mathrm{h})$ & 2.7 & 12 & 2.7 & 14 \\
\hline $\mathrm{CL}_{\mathrm{ECF}, \mathrm{el}}(\mathrm{l} / \mathrm{h})$ & 3.1 & 14 & 3.1 & 18 \\
\hline $\mathrm{V}_{\text {brainECF }}(\mathrm{l})$ & 3.5 & 10 & 3.5 & 12 \\
\hline \multicolumn{5}{|l|}{ Residual error } \\
\hline Plasma & 0.26 & 13 & 0.26 & 14 \\
\hline ECF & 0.51 & 16 & 0.52 & 18 \\
\hline
\end{tabular}

$C L$ clearance, $C V$ coefficient of variation, $k_{m}$ remoxipride concentration at half maximal clearance rate, $Q$ passive distribution between compartments, $R S E$ relative standard error of estimate, $V_{\max }$ maximal clearance rate, $V$ volume of distribution 
a

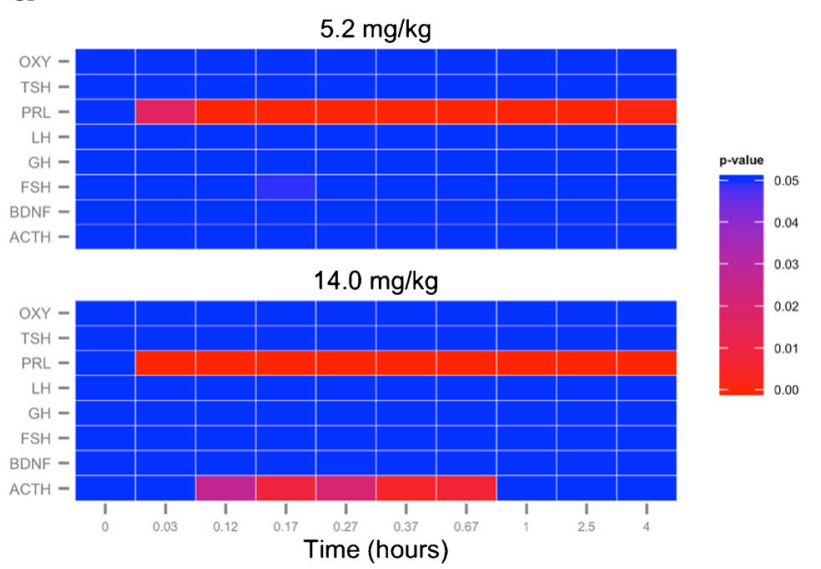

b

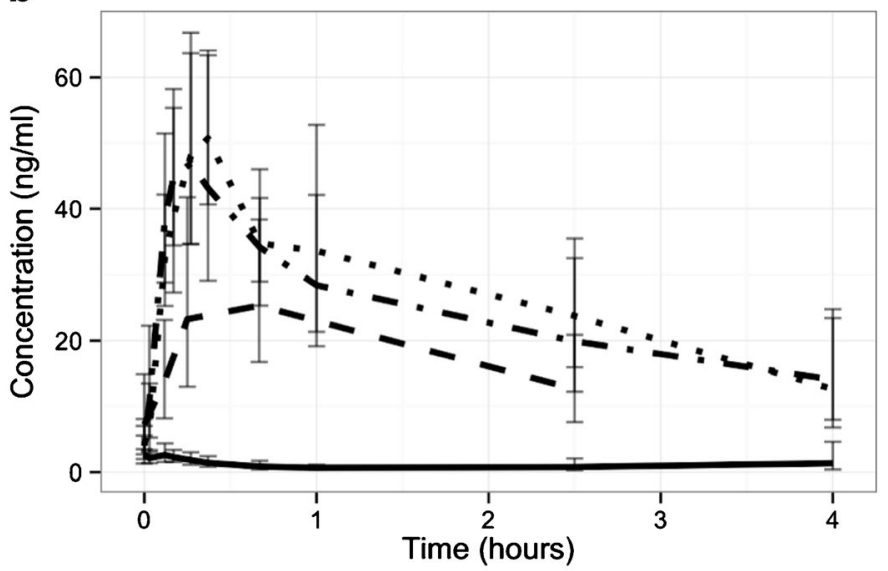

C

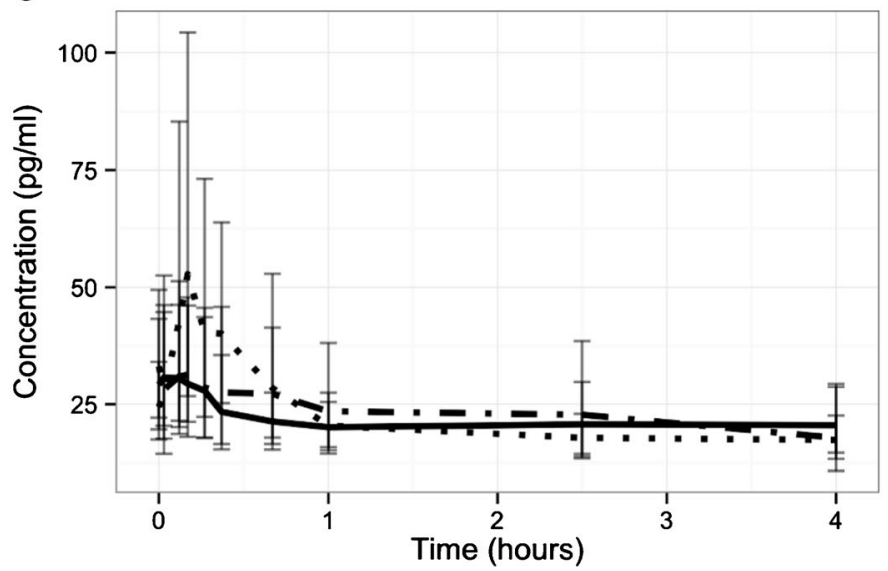

Fig. 2. Biomarker responses upon remoxipride treatment. a Heatmap showing the differential response of the different hormones after 5.2 and $14 \mathrm{mg} / \mathrm{kg}$ remoxipride. Red squares indicate a significant difference compared to the control group that received saline $(p<0.05)$. b Different response time curves for PRL and $\mathbf{c}$ ACTH after 0 (solid), $0.7 \mathrm{mg} / \mathrm{kg}$ (dashed), 5.2 (dot-dashed), or $14 \mathrm{mg} / \mathrm{kg}$ (dotted). Data are geometric mean \pm geometric SD

not after $0.7 \mathrm{mg} / \mathrm{kg}$, PRL showed a two-phasic decline (Fig. 2b). For plasma ACTH, a quick response was observed after $14 \mathrm{mg} / \mathrm{kg}$, with ACTH levels back to baseline within $1 \mathrm{~h}$. No response was observed after $5.2 \mathrm{mg} / \mathrm{kg}$ (Fig. 2c).

\section{PK/PD Model for PRL}

For PRL, a pool model was identified linking brainECF remoxipride to the PRL release, including a positive feedback of PRL on its own synthesis (Fig. 1, upper part). First of all, although PRL in the placebo group initially decreased and subsequently increased (Fig. 2b), this trend was small relative to the pharmacological response $(\sim 3 v s \sim 45 \mathrm{ng} / \mathrm{ml})$. Therefore, a steady baseline was assumed. Second, a turnover model outperformed a pool model as indicated by a significant difference in the OFV (Table III, models A\&B vs C\&D, dOFV $>3.84, d f=1)$. However, inclusion of a positive feedback component of plasma PRL (Fig. 1) on its own synthesis led to a significant improvement in comparison with both a pool model and a turnover model (Table III, models $\mathrm{A}$ and $\mathrm{C} v s$. E dOFV $>7.81, d f=3)$. Moreover, this model explained the two-phasic decline (Fig. 3a), which was not possible without positive feedback (Fig. 3b). The positive feedback parameters ( $E_{\text {max,pf }}$, $\mathrm{EC}_{50, \mathrm{pf}}$ ) were fixed to the values identified by Stevens et al.
(2012), assuming that these system-specific parameters are not different between our earlier and more recent studies. Finally, remoxipride in brainECF could better explain the PRL response than remoxipride in plasma (Table III, model E vs F, dOFV > $3.84, d f=1$ ). Parameter estimates were reasonably precise (RSE $<30 \%$ ), and bootstrap evaluation showed good accuracy (Table IV). The VPC showed a good agreement of the model with the data (Fig. 3a). The model was found to well describe the data from Stevens et al. 2012 (9), although the upper variation was slightly overestimated (Fig. S2).

\section{PK/PD Model for ACTH}

Also, for ACTH, a pool model was identified, linking remoxipride in brainECF to the release of ACTH into plasma (Fig. 1, lower part). First of all, as indicated by a significant difference in the OFV, a pool model performed better than a turnover model (Table II, model A\&B vs C\&D, dOFV >3.84, $d f=1)$. Second, although it was not possible to discriminate between the model with brainECF remoxipride or plasma remoxipride explaining the ACTH response (Table II, model $\mathrm{C} v s \mathrm{D}, \mathrm{dOFV}<3.84, d f=1$ ), the brainECF model (model C) showed better parameter precision (28 vs $89 \%$ ). Moreover, the VPC showed better agreement, albeit a minor difference, 
Table III. Steps in PK/PD Model Development for the Models Describing the PRL and ACTH Responses

\begin{tabular}{|c|c|c|}
\hline Model & Description & OFV \\
\hline \multicolumn{3}{|l|}{ PRL } \\
\hline A & Turnover model with drug effect from remoxipride in brainECF on PRL release & 1861 \\
\hline B & Turnover model with drug effect from remoxipride in plasma on PRL release & 1897 \\
\hline $\mathrm{C}$ & Pool model with drug effect from remoxipride in brainECF on PRL release & 1909 \\
\hline $\mathrm{D}$ & Pool model with drug effect from remoxipride in plasma on PRL release & 1916 \\
\hline $\mathrm{E}$ & $\begin{array}{l}\text { Pool model with drug effect from remoxipride in brainECF on PRL release }+ \text { positive feedback } \\
\text { of PRL in its own synthesis [best model] }\end{array}$ & 1848 \\
\hline \multicolumn{3}{|l|}{ АCTH } \\
\hline A & Turnover model with drug effect ( $\mathrm{E}_{\max }$ model) from remoxipride in ECF on ACTH release & 1957 \\
\hline $\mathrm{B}$ & Turnover model with drug effect ( $\mathrm{E}_{\max }$ model) from remoxipride in plasma on ACTH release & 1869 \\
\hline $\mathrm{C}$ & Pool model with drug effect ( $\mathrm{E}_{\max }$ model) from remoxipride in ECF on ACTH release [best model] & 1862 \\
\hline $\mathrm{D}$ & Pool model with drug effect $\left(\mathrm{E}_{\max }\right.$ model) from remoxipride in plasma on ACTH release & 1861 \\
\hline $\mathrm{E}$ & Pool model with drug effect (linear slope model) from remoxipride in ECF on ACTH release & 1860 \\
\hline
\end{tabular}

with the data for this model (Fig. 3c) as compared to model D, with plasma remoxipride coupled to the response (Fig. 3d). Finally, because ACTH only showed a response after a high remoxipride dose, different drug effect models were compared. An $\mathrm{E}_{\max }$-model showed an equal model fit as a linear slope model (Table III, model D vs E, dOFV <3.84, $d f=1$ ). However, to have a beginning of a clue about the potency of the ACTH response as compared to the PRL response, we sticked to the $\mathrm{E}_{\max }$-model.
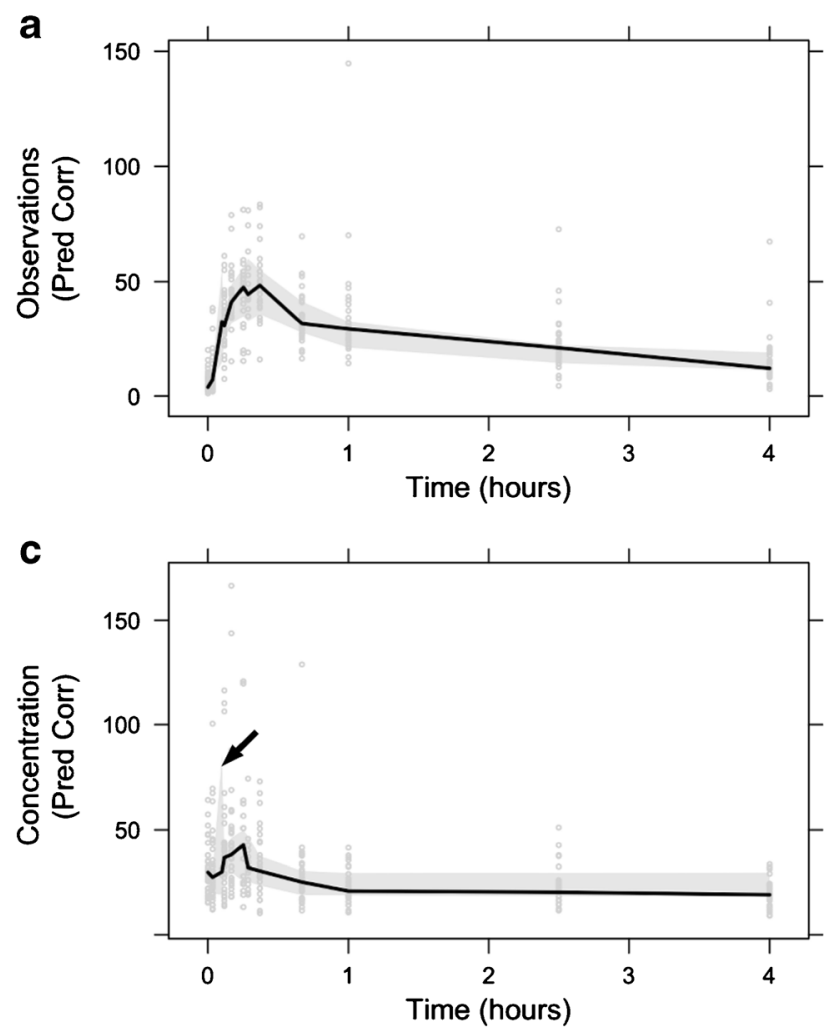

Since the degradation rate of ACTH $\left(\mathrm{k}_{\text {degr,acth }}\right)$ was not identifiable on basis of the current data, it was fixed to a value of $24.5 \mathrm{~h}^{-1}$, as obtained in rats during the ACTH decline after a stress response (23). Also, the maximal drug effect parameter $\left(\mathrm{E}_{\max , \mathrm{rem}, \mathrm{ACTH}}\right)$ was not identifiable. However, because this parameter is a composite of unknown underlying parameters, a literature value was not available. Therefore, $E_{\text {max,rem,ACTH }}$ was chosen on basis of the sensitivity of the OFV to
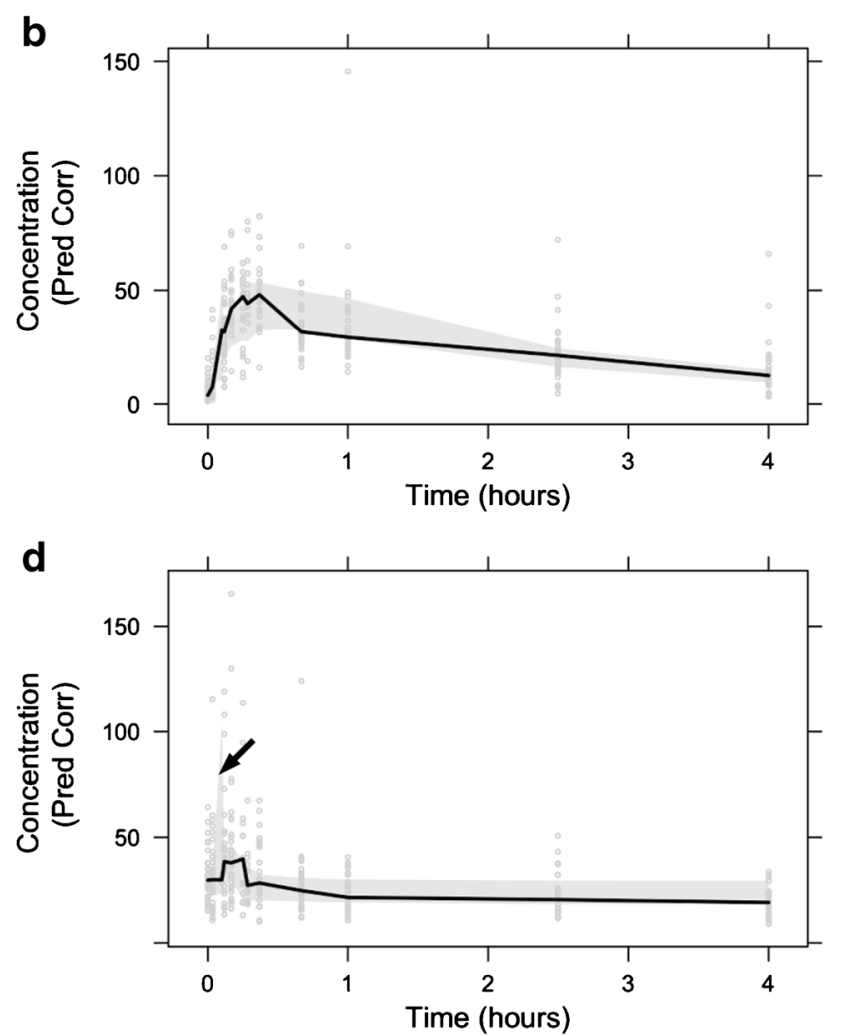

Fig. 3. Prediction corrected visual predictive checks $(n=1000)$ for the remoxipride PK/PD model describing PRL after $0.7,5.2$, and $14 \mathrm{mg} / \mathrm{kg}$ (top) and ACTH after 0, 5.2, and $14 \mathrm{mg} / \mathrm{kg}$ (bottom). The pool models with (a) and without (b) positive feedback are shown for the PRL response. The models with ECF (c) and plasma (d) as target site of action are shown. Solid lines are the observed medians, and the shaded area shows the $95 \%$ prediction interval around the simulated median. The extent to which this area captures the observed median is a measure of correct model specification. Ideally, at each point, $50 \%$ of the prediction interval is above the observed median, and $50 \%$ below. The large prediction intervals in the third bin (see arrow) of figures $\mathbf{c}$ and $\mathbf{d}$ are explained by the fact that this bin contained only two observations 
Table IV. Parameter Estimates and Bootstrap Results for the Remoxipride PK/PD Model Describing the PRL Response

\begin{tabular}{|c|c|c|c|c|}
\hline \multirow[t]{2}{*}{ Parameter } & \multirow[b]{2}{*}{ Parameter estimate } & \multirow[b]{2}{*}{ RSE (\%) } & \multicolumn{2}{|c|}{ Bootstrap $\left(n=100^{\mathrm{c}}\right)$} \\
\hline & & & Bootstrap mean & $\mathrm{CV}(\%)$ \\
\hline $\mathrm{E}_{\text {max,rem,prl }}$ & 13.3 & 12 & 13.8 & 16 \\
\hline $\mathrm{EC}_{50, \mathrm{rem}, \mathrm{prl}}(\mu \mathrm{M})$ & 0.064 & 28 & 0.074 & 63 \\
\hline $\mathrm{Bsl}_{\mathrm{prl}}(\mathrm{ng} \mathrm{ml})$ & 7.3 & 7 & 7.2 & 6 \\
\hline $\mathrm{k}_{\text {synth,prl }}(\mathrm{ng} / \mathrm{ml} / \mathrm{h})$ & $41.7^{\mathrm{a}}$ & & 41.2 & \\
\hline $\mathrm{k}_{\mathrm{rel}, \mathrm{prl}}\left(\mathrm{h}^{-1}\right)$ & 0.35 & 12 & 0.36 & 18 \\
\hline $\mathrm{k}_{\mathrm{degr}, \mathrm{prl}}\left(\mathrm{h}^{-1}\right)$ & $5.72^{\mathrm{b}}$ & & 5.72 & \\
\hline $\mathrm{E}_{\text {max,pf,prl }}$ & $3.47^{\mathrm{b}}$ & & 3.47 & \\
\hline $\mathrm{EC}_{50, \mathrm{pf}, \mathrm{prl}}(\mathrm{ng} / \mathrm{ml})$ & $12.4^{\mathrm{b}}$ & & 12.4 & \\
\hline \multicolumn{5}{|l|}{ IIV } \\
\hline $\mathrm{Bsl}_{\mathrm{prl}}$ & 0.075 & 19 & 0.071 & 43 \\
\hline \multicolumn{5}{|l|}{ Residual error } \\
\hline Proportional & 0.13 & 8 & 0.13 & 13 \\
\hline
\end{tabular}

$B s l$ baseline, $E_{\max }$ maximal effect, $C V$ coefficient of variation, $E C_{50}$ drug concentration at half maximal effect, $I I V$ interindividual variability, $k_{\text {synth }}$ PRL synthesis rate in the lactotroph, $k_{r e l}$ PRL release rate from the lactotroph to plasma, $k_{\text {degr }}$ degradation rate of PRL in plasma, $p f$ positive feedback, $\mathrm{prl}$ prolactin, $r e m$ remoxipride, RSE relative standard error of estimate

${ }^{a} \mathrm{k}_{\text {synth }}$ was calculated from bsl $\mathrm{prl} * \mathrm{k}_{\mathrm{degr}}$,prl

${ }^{b}$ Values were obtained from literature

${ }^{c} 100$ out of 100 bootstrap runs minimized successfully

$\mathrm{E}_{\text {max,rem,ACTH }}$ values of $1,7.5,10,15,20$, and 100. Up till a value of 10 , the OFV dropped significantly, but from a value of 10 or higher, it showed no significant difference (dOFV <3.84). Therefore, $E_{\text {max,rem,acth }}$ was fixed to 10. Here, it must be noted that, with changing $\mathrm{E}_{\max , \mathrm{rem}, \mathrm{ACTH}}$, the $\mathrm{EC}_{50}$ changed almost linearly with the change in $\mathrm{E}_{\max }$, while other parameters remained the same. Parameter estimates of the best model (Table III, model C) showed good precision (RSE $<30 \%$ ) and accuracy (Table V), and the VPC showed good agreement between the model and the data (Fig. 3c).

\section{DISCUSSION}

Our goal was to apply the multi-biomarker discovery approach in a quantitative manner, with remoxipride as a paradigm compound. To that end, hormones from the neuroendocrine system were analyzed as a source for a multi-biomarker to represent remoxipride effect. We showed that not only PRL, but also ACTH acts as biomarker for remoxipride pharmacology. Both these hormones are synthesized in the so-called "troph" cells in the anterior pituitary, from which they are continuously released. In our study, we

Table V. Parameter Estimates and Bootstrap Results for the Remoxipride PK/PD Model Describing the ACTH Response

\begin{tabular}{|c|c|c|c|c|}
\hline \multirow[t]{2}{*}{ Parameter } & \multirow[b]{2}{*}{ Parameter estimate } & \multirow[b]{2}{*}{ RSE (\%) } & \multicolumn{2}{|c|}{ Bootstrap $\left(n=100^{\mathrm{d}}\right)$} \\
\hline & & & Bootstrap mean & $\mathrm{CV}(\%)$ \\
\hline $\mathrm{E}_{\text {max,rem,ACTH }}$ & $10^{\mathrm{a}}$ & & 10 & \\
\hline $\mathrm{EC}_{50, \text { rem, }}$ АСтH $(\mu \mathrm{M})$ & 2.61 & 19 & 2.66 & 30 \\
\hline $\mathrm{Bsl}_{\mathrm{ACTH}}(\mathrm{pg} / \mathrm{ml})$ & 24.6 & 8 & 24.6 & 5 \\
\hline $\mathrm{k}_{\text {synth,АCTH }}(\mathrm{pg} / \mathrm{ml} / \mathrm{h})$ & $603^{\mathrm{b}}$ & & 603 & \\
\hline $\mathrm{k}_{\mathrm{rel}, \mathrm{ACTH}}\left(\mathrm{h}^{-1}\right)$ & 3.13 & 18 & 3.03 & 21 \\
\hline $\begin{array}{l}\mathrm{k}_{\text {degr,ACTH }}\left(\mathrm{h}^{-1}\right) \\
\text { IIV }\end{array}$ & \multicolumn{4}{|c|}{ IIV } \\
\hline $\mathrm{Bsl}_{\mathrm{prl}}$ & 0.15 & 26 & 0.14 & 26 \\
\hline \multicolumn{5}{|l|}{ Residual error } \\
\hline Proportional & 0.12 & 5 & 0.12 & 17 \\
\hline
\end{tabular}

$B s l$ baseline, $E_{\max }$ maximal effect, $C V$ coefficient of variation, $E C_{50}$ drug concentration at half maximal effect, $I I V$ interindividual variability, $k_{\text {synth }}$ ACTH synthesis rate in the lactotroph, $k_{\text {rel }}$ ACTH release rate from the lactotroph to plasma, $k_{\text {degr }}$ degradation rate of ACTH in plasma, $p f$ positive feedback, $p r l$ ACTH, rem remoxipride, $R S E$ relative standard error of estimate

${ }^{a}$ Parameter was not identifiable, and the value was chosen on basis of a sensitivity analysis. Informative over terminated

${ }^{b} \mathrm{k}_{\text {synth }}$ was calculated from bsl prl $^{*} \mathrm{k}_{\text {degr,prl }}$

${ }^{c}$ Value was obtained from literature

${ }^{d} 89$ out of 100 bootstrap runs minimized successfully 
quantified the effect of remoxipride on the PRL and ACTH release, as described by pool models, thereby gaining a comprehensive understanding of remoxipride effect on the neuroendocrine system. With that, we revealed the in vivo concentration effect relation of brainECF remoxipride with the hormones PRL and ACTH enabling the prediction of a neuroendocrine response for other doses of remoxipride.

\section{Pharmacokinetics}

The brainECF concentrations were described by a threecompartment PK model, which was developed first to be subsequently linked to the PK/PD model. This model included drug elimination from the brain, confirming a previously developed PK model for remoxipride in rats (22). Whether this represented remoxipride active transport from brainECF to plasma, metabolism of remoxipride in the brain or efflux of remoxipride from brainECF to cerebrospinal fluid (CSF) remains to be answered. No studies on remoxipride being a substrate for efflux transporters have been reported, to the knowledge of the authors. Interestingly, O-demethylase activity has been observed in the rat brain (24), suggesting that remoxipride metabolism in the brain may have occurred. Furthermore, whereas Stevens et al. (2011) found linear elimination from plasma, we identified saturable elimination, described by Michaelis Menten kinetics. They applied a 30min infusion time, but we used a 10-min infusion time with similar dose levels. This led to a 1.5 times higher maximal plasma remoxipride concentrations in the current study, making it more likely to observe saturation of metabolic clearance. This is not surprising for remoxipride since in rats, it is eliminated mainly through liver enzymatic processes of demethylation and aromatic hydroxylation (25). However, in humans, remoxipride is metabolized mainly via oxidation (25), which may not be saturated at these concentrations. Indeed, linear elimination was identified for remoxipride in humans (26).

\section{Pharmacodynamics}

Dopaminergic control over the neuroendocrine system is established via the tuberoinfundibular system, which consists of TIDA, THDA, and periventricular hypothalamic dopaminergic (PHDA) neurons that connect the hypothalamus to the pituitary (13). Therefore, we were interested in plasma hormones that are released from the AL or the PL of the pituitary as a reflection of central pharmacology of dopaminergic agents. Whereas PRL has been used as a biomarker for central dopaminergic activity, exerted via the tuberoinfundibular system, other neuroendocrine hormones have not been used for this purpose. Surprisingly, despite the potential to respond to dopaminergic perturbation as described in the introduction, FSH, LH, GH, TSH, and BDNF did not show a response to a single dose of remoxipride. Thus, a D2 agonistic effect on these hormones or presence of the $\mathrm{D} 2$ receptor on the secretory cells does not necessarily imply a response to dopamine D2 antagonism. Also, within $4 \mathrm{~h}$, we could not identify feedback regulation on these five hormones by the two responding hormones. Unfortunately, we were not able to measure OXT since its levels were lower than the limit of quantification, although it is likely that there is an interaction between PRL and OXT (12). However, using the multi-biomarker discovery approach on eight hormones, we identified not only PRL, but also ACTH as a biomarker for the $\mathrm{D} 2$ antagonist remoxipride.

\section{PK/PD Model for PRL}

To use PRL as a biomarker for prediction of the response after a second remoxipride administration, it is important to take into account underlying tolerance mechanisms, either being the depletion of the lactotroph (pool model) $(8,9)$ or the stimulation of dopamine production by PRL (agonist-antagonist interaction model) (27). Although both models were able to account for the tolerance, only the pool model could explicitly separate drug-specific and systemspecific parameters, enabling a proper translational step from animal to human (9). The models seem to have similar flexibility to fit the prolactin response after a D2 antagonist challenge (27-29), and we also found a similar goodness-of-fit between the pool and the AAI models (AIC 1860 versus AIC 1844). In fact, to be able to discriminate between these models, a continuous infusion with a D2 antagonist should be applied. According to the pool model, the plasma PRL concentrations will drop to baseline after the pool is empty. Following the AAI model, the plasma PRL concentrations will reach a higher steady state during the continuous exposure. In this study, a pool model was found to describe the prolactin response with brainECF remoxipride related to the release of PRL. Furthermore, in agreement with Stevens et al. (2012), the inclusion of a positive feedback of PRL on its own synthesis significantly improved the model with particularly the two-phasic decline being better described (Fig. 3b). This feedback mechanism works by increasing the "refilling" of the lactotrophs after release of PRL, possibly mediated through stimulation of the PRL receptor on the lactotrophs $(9,10)$. Although the inclusion of the positive feedback improved the data fitting, caution must be taken with this type of non-linear models. Bakshi et al. (2016) published a tutorial on mathematical analysis of ordinary differential equation (ODE) model behavior, showing that the pool model with positive feedback has two steady states and interesting stability behavior. This means that the model may converge to one or the other steady state, depending on the specific simulated trajectory, which could be affected by small changes in dose or parameter values. Such behavior is not physiological, and therefore caution should be taken when extrapolating to other parameter regions or dosages. Still, the model proved powerful in translating the PRL response from animal to human, which provided inclusion of an if-condition that forced the prolactin concentrations to remain above baseline prolactin concentrations $(9,30)$. Simulations with the current model including the ifcondition up to $10 \mathrm{~h}$ showed a convergence to the correct baseline.

Furthermore, dopamine D2 receptors influence PRL release both at the level of the hypothalamus (TIDA neurons) and the pituitary (lactotrophs) (10). Stimulation of PRL release via D2 receptor antagonism at the TIDA neurons would be mediated through reduced dopamine release into the pituitary, whereas stimulation via the lactotrophs would be mediated through antagonism directly at the level of the pituitary. Reduced dopamine release via D2 antagonism at the TIDA neurons is driven by remoxipride in 
brainECF, whereas D2 antagonism at the lactotrophs is driven by remoxipride in plasma, since the pituitary is exposed to blood. With data on remoxipride both in plasma and brainECF, we could compare these hypotheses to find that PRL release was driven by remoxipride in brainECF (Table III). However, domperidone, another D2 antagonist which hardly penetrates the brain, also stimulates prolactin release (31). Indeed, drugs with limited brain penetration exhibit a low $\mathrm{ED}_{50}$ of $\mathrm{D} 2$ receptor occupancy in the pituitary as compared to that in the striatum, which correlated with the $\mathrm{ED}_{50}$ of prolactin release (peripheral effect) as compared to $\mathrm{ED}_{50}$ of apomorphine-induced stereotype behavior (central effect) (32). This suggests that direct antagonism of pituitary $\mathrm{D} 2$ receptors is responsible for the prolactin release. Unfortunately, no such data exists on D2 antagonists with high brain penetration $\left(\mathrm{k}_{\mathrm{p}, \mathrm{uu}}>1\right)$ to investigate whether the reduced dopamine release in the pituitary will become dominant in stimulating the prolactin release. On basis of temporal PK/PD analysis, we found brainECF remoxipride driving the PRL response. Therefore, because remoxipride highly penetrates the brain $\left(\mathrm{k}_{\mathrm{p}, \mathrm{uu}} \sim 1\right)$, it is suggested that for D2 antagonists with high brain penetration, the reduced dopamine release into the pituitary becomes dominant in driving the PRL response.

Finally, the estimated $\mathrm{EC}_{50, \text { rem,prl }}$ of $64 \mathrm{nM}$ was 2-4-fold different from in vitro binding potencies of $113 \mathrm{nM}$ (33) and $240 \mathrm{nM}$ (34) for remoxipride on striatal D2 receptors measured in rat brain homogenate, but comparable to the $\mathrm{EC}_{50}$ of $80 \mathrm{nM}$ found for the previously developed pool model on basis of in vivo data (9). The discrepancy between in vivo and in vitro estimates might be explained by a role of active metabolites (25). Although their concentrations in plasma are 10-1000 times lower compared to remoxipride (25), four metabolites (FLA797, FLA908, NCQ436, NCQ469) showed 2-200 times higher in vitro affinity for the D2 receptor than the parent remoxipride (33). Moreover, these metabolites showed in vivo activity on DOPA accumulation in rat striatum (35). Interestingly, this could be an alternative explanation of the two-phasic decline of prolactin (Fig. 2b). In that case, the PRL response would be the consequence of D2 receptor binding of both remoxipride and its active metabolites. Since the PK profile of the active metabolite lags behind that of remoxipride (25), the first part of the PRL response would be explained by remoxipride effect, whereas the second part would be driven by the active metabolites. Nevertheless, the contribution of active metabolites to the in vivo remoxipride potency is tentative and in further research remoxipride should be analyzed in conjunction with its active metabolites to obtain a complete picture of its $\mathrm{PK} / \mathrm{PD}$ characteristics.

\section{ACTH as a Biomarker of Adrenergic Receptor Antagonism}

To the knowledge of the authors, no studies have been performed for the ACTH response upon remoxipride, but other dopaminergic agents have been investigated. The relation between dopaminergic agents and $\mathrm{ACTH}$ is rather intriguing. Although the dopamine D2 agonist bromocriptine is used to treat ACTH hypersecretion (14), suggesting an inhibiting effect, this appears to be specific for tumor cells (36). On the contrary, ACTH release in rats was stimulated by the dopamine D2 agonists quinpirole and apomorphine, as well as by the dopamine reuptake inhibitor GBR12909. These effects were blocked by the dopamine D2 antagonists haloperidol and sulpiride suggesting a D2-specific response $(37,38)$. However, counterintuitively, haloperidol could also stimulate ACTH release $(39,40)$, which was also observed for the D2 antagonists thioproperazine (41) and eticlopride (42). Yet, sulpiride did not stimulate ACTH release (37).

Since the stimulatory effect of D2 agonists on ACTH release has been confirmed to be D2 specific, it is likely that the effect of the D2 antagonists is an off-target effect that these drugs have in common.

Although 5- $\mathrm{HT}_{1 \mathrm{~A}}$ agonists can induce ACTH release (43), remoxipride and haloperidol have no affinity for the 5$\mathrm{HT}_{1}$ receptors (34). Moreover, 5- $\mathrm{HT}_{1 \mathrm{~A}}$ agonists are known to stimulate GH release (43), which was not observed for remoxipride in the present study. On the other hand, the in vitro affinity to adrenergic receptors of both remoxipride (to $\alpha 2$ receptor) and haloperidol (to $\alpha 1$ receptor) was only 10-50 times lower than to dopamine $\mathrm{D}_{2}$ receptors $(34,44)$. Maximal ECF remoxipride concentrations that were observed in rats receiving $14.0 \mathrm{mg} / \mathrm{kg}(1.3-3.1 \mu \mathrm{M})$ exceed the $\mathrm{EC}_{50, \mathrm{prl}}(0.064 \mu \mathrm{M})$ by $20-50$ times. In contrast, sulpiride has negligible affinity to adrenergic receptors (at least 100 times lower than its affinity to $\mathrm{D}_{2}$ receptor) $(34,44)$. In addition, haloperidol showed considerable in vivo receptor occupancy and functional activity at central adrenergic receptors $(45,46)$. The endogenous ligands for adrenergic receptors are epinephrine and norepinephrine, which are known to inhibit the release of corticotropin releasing hormone $(\mathrm{CRH})$ from the hypothalamus to the pituitary (47). In the pituitary, $\mathrm{CRH}$ stimulates the release of ACTH from the corticotrophs, which was found to be the pathway for thioproperazine to stimulate ACTH release (41). This is in line with our observation that ACTH release is likely to be linked to remoxipride in brainECF, suggesting a drug-receptor interaction at the hypothalamus level. Following these observations, it is thus well possible that the effect of remoxipride on ACTH is elicited via antagonism of the centrally located adrenergic receptor, leading to an increased release of CRH and ACTH.

\section{PK/PD Model for ACTH}

The mechanism of ACTH being released upon remoxipride administration thus seems similar to that of PRL, albeit via another pathway. Whereas PRL is released from lactotrophs, stimulated by reduction of dopamine, ACTH is secreted from corticotrophs, stimulated by induction of $\mathrm{CRH}$. This provides further evidence for our observation that a pool model described the ACTH response (Table III). Furthermore, as discussed above, ACTH is suggested as a biomarker for an adrenergic response. Although it was not possible to determine the exact $\mathrm{EC}_{50, \mathrm{ACTH}}(2.61 \mu \mathrm{M})$, since it was dependent on the $\mathrm{E}_{\max , \mathrm{ACTH}}$ that was fixed to an arbitrary value of at least 10 , we can conclude that it is at least 40 times higher than $\mathrm{EC}_{50 \text {,prl }}(0.064 \mu \mathrm{M})$. This confirms the earlier suggestion that ACTH represents an offtarget effect of remoxipride, possibly via the adrenergic receptor.

Thus, by simultaneous analysis of eight hormones in plasma for a multi-biomarker approach, we could identify ACTH as additional biomarker for remoxipride in rats. Together with PRL, this biomarker provides insights into the effects of remoxipride on the neuroendocrine system 
through different pathways. However, whereas animals in preclinical experiments are very similar, having the same genetic background and environmental conditions, humans are highly heterogeneous in terms of genetic background, lifestyle, disease, age, and other factors that may influence drug efficacy (48). Such variation may impact the response of the neuroendocrine system to D2 antagonists. For example, corticotrophs in pituitary tumors have higher D2 receptor expression and become sensitive to dopamine D2 agents (36), which would impact the ACTH response, but not the PRL response. Thus, taking into account a multi-biomarker would give more precise insights in the PK/PD processes on an individual level, providing opportunities for personalized medicine.

Furthermore, as discussed above, the D2 antagonists haloperidol (40), thioproperazine (41), eticlopride (42), and remoxipride all stimulate ACTH release, whereas sulpiride (37) does not show such response. With PRL only, it is shown that some D2 antagonists cause hyperprolactinemia, but others not, providing a sub-classification of D2 antagonists. A multi-biomarker provides further sub-classification, for example distinguishing sulpiride from haloperidol, thioproperazine, eticlopride, and remoxipride. Thus, our approach enables detailed sub-classification and provides comprehensive insights in differential effects among drugs. As such, the approach should ultimately be applied to a series of dopamine $\mathrm{D}_{2}$ antagonists and agonists.

Finally, since now, we could reveal the neuroendocrine response by combining a multi-biomarker discovery approach with PK/PD modeling, we have provided a conceptual basis to use for example metabolism for a multi-biomarker paradigm. This is expected to provide a much more extensive multi-biomarker than the biomarkers used in the current study. With the metabolomics technique, more than 100 endogenous metabolites can be measured, providing a promising next step with an untargeted biomarker discovery approach rather than the relatively targeted approach exploited in our study. These metabolite responses are then analyzed by multivariate statistics (49), which would identify the underlying shared responses among the metabolites and provide a multi-biomarker. Metabolomics has been successfully applied for identification of new biomarkers of drug effects (2-4), although no studies have been performed that combined it with PK/PD modeling. Therefore, further studies should expand on the current study by using metabolomics for untargeted biomarker discovery.

In conclusion, often multi-biomarker discovery and PK/ PD modeling are separated fields, limiting the insights that can be obtained in in vivo pharmacological studies. In our study, we overcame this limitation by combining these fields, revealing remoxipride effects not only on PRL, but also ACTH. Moreover, using PK/PD modeling, we revealed that both hormone responses were (i) likely to be driven by remoxipride in brainECF and (ii) described by a pool model according to the underlying physiology of hormone release from "troph" cells. With that, we quantified the dose response over a large dose range, enabling the prediction of neuroendocrine responses after different doses of remoxipride. More generally speaking, our study shows how multi-biomarker discovery can reveal and PK/PD modeling can quantify the multiple neuroendocrine responses for single drug action.

\section{ACKNOWLEDGMENTS}

The authors thank Shionogi \& Co., LTD, Japan for their financial support and Shuichi Ohnishi and colleagues for the fruitful discussions. This work has also received support from the EU/EFPIA Innovative Medicines Initiative (IMI) Joint Undertaking, "Kinetics for Drug Discovery," K4DD (grant no. 115366). The authors thank Robin Hartman for performing surgeries contributing to the experiments, Rob van Wijk for contributing to the experiments, and Dirk-Jan van den Berg for contributing to the remoxipride and hormone analysis.

Authors' contribution EL and WB designed the WB02/ WB06 study; YW designed the EW01 study; WB and YW performed animal experiments; WB and YW performed the remoxipride and hormone analysis; $\mathrm{WB}$ and $\mathrm{BG}$ did the data analysis. EL and PG provided overall supervision of the study.

\section{COMPLIANCE WITH ETHICAL STANDARDS}

Conflict of interest The authors have no interest to declare.

Open Access This article is distributed under the terms of the Creative Commons Attribution 4.0 International License (http://creativecommons.org/licenses/by/4.0/), which permits unrestricted use, distribution, and reproduction in any medium, provided you give appropriate credit to the original author(s) and the source, provide a link to the Creative Commons license, and indicate if changes were made.

\section{REFERENCES}

1. Van Der Greef J, Martin S, Juhasz P, Adourian A, Plasterer T, Verheij ER. The art and practice of systems biology in medicine : mapping patterns of relationships. J Proteome Res. 2007;6:1540-59.

2. Quinones MP, Kaddurah-Daouk R. Metabolomics tools for identifying biomarkers for neuropsychiatric diseases. Neurobiol Dis. Elsevier B.V.; 2009;35 Suppl 2:165-76.

3. Van Der Greef J, Mcburney RN. Rescuing drug discovery: in vivo systems pathology and systems pharmacology. Nat Rev Drug Discov. 2005;4:961-8.

4. Kaddurah-Daouk R, Kristal BS, Weinshilboum RM. Metabolomics: a global biochemical approach to drug response and disease. Annu Rev Pharmacol Toxicol. 2008;48:653-83.

5. Danhof M, Alvan G, Dahl SG, Kuhlmann J, Paintaud G. Mechanism-based pharmacokinetic-pharmacodynamic modeling-a new classification of biomarkers. Pharm Res. 2005;22(9):1432-7.

6. Morgan P, Van Der Graaf PH, Arrowsmith J, Feltner DE, Drummond KS, Wegner CD, et al. Can the flow of medicines be improved? Fundamental pharmacokinetic and pharmacological principles toward improving Phase II survival. Drug Discov Today. Elsevier Ltd; 2012;17 Suppl 9/10:419-24.

7. de Lange ECM, Ravenstijn PGM, Groenendaal D, van Steeg TJ. Toward the prediction of CNS drug-effect profiles in physiological and pathological conditions using microdialysis and mechanism-based pharmacokinetic-pharmacodynamic modeling. AAPS J. 2005;7(3):E532-43.

8. Movin-Osswald G, Hammarlund-Udenaes M. Prolactin release after remoxipride by an integrated pharmacokineticpharmacodynamic model with intra- and interindividual aspects. J Pharmacol Exp Ther. 1995;274:921-7. 
9. Stevens J, Ploeger BA, Hammarlund-Udenaes M, Osswald G, Van Der Graaf PH, Danhof M, et al. Mechanism-based PK-PD model for the prolactin biological system response following an acute dopamine inhibition challenge: quantitative extrapolation to humans. J Pharmacokinet Pharmacodyn. 2012;39:463-77.

10. Freeman ME, Kanyicska B, Lerant A, Nagy G. Prolactin : structure, function, and regulation of secretion. Physiol Rev. 2000;80(4):1523-631.

11. Petty RG. Prolactin and antipsychotic medications: mechanism of action. Schizophr Res. 1999;35:67-73.

12. Kennett JE, McKee DT. Oxytocin: an emerging regulator of prolactin secretion in the female rat. J Neuroendocrinol. 2012;24(3):403-12.

13. Lerant A, Herman ME, Freeman ME. Dopaminergic neurons of periventricular and arcuate nuclei of pseudopregnant rats: semi circadian rhythm in fos-related antigen immunoreactivity and in dopamine concentration. Endocrinology 1996;137 Suppl 9.

14. Mercado-Asis LB, Yanovski JA, Tracer HL, Chik CL, Cutler GB. Acute effects of bromocriptine, cyproheptadine, and valproic acid on plasma adrenocorticotropin secretion in Nelson's syndrome. J Endocrinol Metab. 1997;82(2):514-7.

15. Howlett TA, Willis D, Walker G, Wass JAH, Trainer PJ. Control of growth hormone and IGF1 in patients with acromegaly in the UK: responses to medical treatment with somatostatin analogues and dopamine agonists. Clin Endocrinol (Oxf). 2013;79(5):689-99.

16. Ishibashi M, Yamaji T. Direct effects of catecholamines, thyrotropinreleasing hormone, and somatostatin on growth hormone and prolactin secretion from adenomatous and nonadenomatous human pituitary cells in culture. J Clin Invest. 1984;73(1):66-78.

17. Gatto F, Barbieri F, Gatti M, Wurth R, Schulz S, Ravetti JL, et al. Balance between somatostatin and $\mathrm{D} 2$ receptor expression drives TSH-secreting adenoma response to somatostatin analogues and dopastatins. Clin Endocrinol (Oxf). 2012;76(3):407-14.

18. Winters SJ, Ghooray DT, Yang RQ, Holmes JB, O'Brien AR, Morgan J, et al. Dopamine-2 receptor activation suppresses PACAP expression in gonadotrophs. Endocrinology. 2014;155(7):2647-57.

19. Westerhout J, Ploeger B, Smeets J, Danhof M, Lange ECM. Physiologically based pharmacokinetic modeling to investigate regional brain distribution kinetics in rats. AAPS J. 2012;14(3):543-53.

20. Stevens J, Suidgeest E, Van Der Graaf PH, Danhof M, De Lange ECM. A new minimal-stress freely-moving rat model for preclinical studies on intranasal administration of CNS drugs. Pharm Res. 2009;26(8):1911-7.

21. Stevens J, van den Berg DJ, de Ridder S, Niederländer HAG, van der Graaf PH, Danhof M, et al. Online solid phase extraction with liquid chromatography-tandem mass spectrometry to analyze remoxipride in small plasma-, brain homogenate-, and brain microdialysate samples. J Chromatogr B. 2010;878(13/14):969-75.

22. Stevens J, Ploeger BA, Van Der Graaf PH, Danhof M, De Lange ECM. Systemic and direct nose-to-brain transport pharmacokinetic model for remoxipride after intravenous and intranasal administration. Drug Metab Dispos. 2011;39:2275-82.

23. Matsuyama H, Ruhmann-Wennhold A, Johnson LR, Nelson DH. Disappearance rates of exogenous and endogenous ACTH from rat plasma measured by bioassay and radioimmunoassay. Metabolism. 1972;21(1):30-5.

24. Jolivalt C, Minn A, Vincent-Viry M, Galteau M-M, Siest G. Dextromethorphan O-demethylase activity in rat brain microsomes. Neurosci Lett. 1995;187:65-8.

25. Widman M, Nilsson L, Bryske B, Lundström J. Disposition of remoxipride in different species. Drug Res (Stuttg). 1993;43(1):287-97.

26. Movin-Osswald G, Boelaert J, Hammarlund-Udenaes M, Nilsson LB. The pharmacokinetics of remoxipride and metabolites in patients with various degrees of renal function. $\mathrm{Br} \mathrm{J}$ Clin Pharmacol. 1993;35:615-22.

27. Ma G, Friberg LE, Movin-Osswald G, Karlsson MO. Comparison of the agonist-antagonist interaction model and the pool model for the effect of remoxipride on prolactin. Br J Clin Pharmacol. 2010;70(6):815-24.

28. Friberg LE, Vermeulen AM, Petersson KJF, Karlsson MO. An agonist-antagonist interaction model for prolactin release following risperidone and paliperidone treatment. Clin Pharmacol Ther. 2009;85(4):409-17.
29. Taneja A, Vermeulen A, Huntjens DRH, Danhof M, De Lange ECM, Proost JH. A comparison of two semi-mechanistic models for prolactin release and prediction of receptor occupancy following administration of dopamine D2 receptor antagonists in rats. Eur J Pharmacol. 2016; Elsevier.

30. Bakshi S, de Lange E, van der Graaf P, Danhof M, Peletier L. Understanding the behavior of systems pharmacology models using mathematical analysis of differential equations: prolactin modeling as a case study. CPT Pharmacometrics Syst Pharmacol. 2016;5(7):339-57.

31. Kiem DT, Nagy GM, Barna I, Makara GB. Domperidone stimulates prolactin secretion in rats with complete destruction of the mediobasal hypothalamus. Brain Res Bull. 1997;44(2):151-4.

32. Kapur S, Langlois X, Vinken P, Megens AAHP, De Coster R, Andrews JS. The differential effects of atypical antipsychotics on prolactin elevation are explained by their differential bloodbrain disposition: a pharmacological analysis in rats. J Pharmacol Exp Ther. 2002;302(3):1129-34.

33. Mohell N, Sällemark M, Rosqvist S, Malmberg A, Högberg T, Jackson DM. Binding characteristics of remoxipride and its metabolites to dopamine D2 and D3 receptors. Eur J Pharmacol. 1993;238:121-5.

34. Köhler C, Hall H, Magnusson O, Lewander T, Gustafsson K. Biochemical pharmacology of the atypical neuroleptic remoxipride. Acta Psychiatr Scand Suppl. 1990;358:27-36.

35. Ahlenius S, Ericson E, Hillegaart V, Nilsson LB, Salmi P, Wijkström A. In vivo effects of remoxipride and aromatic ring metabolites in the rat. J Pharmacol Exp Ther. 1997;283(3):1356-66.

36. Engler D, Redei E, Kola I. The corticotropin-release inhibitory factor hypothesis: a review of the evidence for the existence of inhibitory as well as stimulatory hypophysiotropic regulation of adrenocorticotropin secretion and biosynthesis. Endocr Rev. 1999;20(4):460-500.

37. Borowsky B, Kuhn CM. D1 and D2 dopamine receptors stimulate hypothalamo-pituitary-adrenal activity in rats. Neuropharmacology. 1992;31(7):671-8.

38. Ježová D, Jurčovičová J, Vigaš M, Murgaš K, Labrie F. Increase in plasma ACTH after dopaminergic stimulation in rats. Psychopharmacology (Berlin). 1985;85(2):201-3.

39. Borowsky B, Kuhn CM. Monoamine mediation of cocaineinduced hypothalamo-pituitary-adrenal activation. J Pharmacol Exp Ther. 1991;256(1):204-10.

40. Giraud P, Lissitzky JC, Gillioz P, Oliver C. Influence of Haloperidol on ACTH and B-endorphin secretion in the rat. Eur J Pharmacol. 1980;62:1976-8.

41. Proulx-Ferland L, Meunier H, Cote J, Dumont D, Gagne B, Labrie F. Multiple factors involved in the control of ACTH and alpha-MSH secretion. J Steroid Biochem. 1983;19(1B):439-45.

42. Belda X, Armario A. Dopamine D1 and D2 dopamine receptors regulate immobilization stress-induced activation of the hypothalamus-pituitary-adrenal axis. Psychopharmacology (Berlin). 2009;206(3):355-65.

43. de Koning P, de Vries MH. A comparison of the neuroendocrinological and temperature effects of DU 29894, flesinoxan, sulpiride and haloperidol in normal volunteers. Br J Clin Pharmacol. 1995;39(1):7-14.

44. Hyttel J. SCH 23390-The first selective dopamine D-1 antagonist. Eur J Pharmacol. 1983;91(1):153-4.

45. Schotte A, Janssen PFM, Gommeren W, Luyten W, VanGompel P, Lesage AS, et al. Risperidone compared with new and reference antipsychotic drugs. Psychopharmacology (Berlin). 1996;124:57-73.

46. Sanchez C, Arnt J, Dragsted N, Hyttel J, Lembol H, Meier E, et al. Neurochemical and in vivo pharmacological profile of sertindole, a limbic-selective neuroleptic compound. Drug Dev Res. 1991;22:239-50.

47. Weiner RI, Ganong WF. Role of brain monoamines and histamine in regulation of anterior pituitary secretion. Physiol Rev. 1978;58(4):905-76.

48. de Lange EC. The mastermind approach to CNS drug therapy: translational prediction of human brain distribution, target site kinetics, and therapeutic effects. Fluids Barriers CNS. 2013;10(1):12.

49. Xia J, Sinelnikov IV, Han B, Wishart DS. MetaboAnalyst 3.0making metabolomics more meaningful. Nucleic Acids Res. 2015;43(W1):W251-7. 